\title{
Politiques et Modèles d'Innovation en Afrique : Enjeux systémiques et structurels par le renforcement des Capacités
}

\author{
Innovation Policies and Models in Africa: Systemic and Structural \\ Challenges through Capacity Building
}

\author{
Ludovic Temple ${ }^{1,2}$, Vanessa Casadella ${ }^{3}$ \\ ${ }^{1}$ CIRAD, UMR Innovation, TA C-85 / 15, 73 rue Jean-François Breton 34398 Montpellier Cedex 5, Montpellier, France \\ ${ }^{2}$ INNOVATION, Université de Montpellier, CIRAD, INRA, Montpellier SupAgro, Montpellier, France \\ ${ }^{3}$ Université Picardie Jules Verne, CRIISEA, Vanessa.casadella@u-picardie.fr
}

RÉSUMÉ. Cet article introductif part du renouvellement des enjeux du développement en Afrique au regard de l'accroissement des inégalités, du changement climatique, de l'insécurité alimentaire, de la croissance démographique, des conflits politiques pour interpeller l'efficacité des politiques publiques. II interroge, dans ce contexte, comment adapter un cadre d'analyse systémique et interactif de l'innovation pour renforcer les capacités sociétales vectrices de nouvelles trajectoires technologiques, économiques et sociales. Cette proposition met ainsi en exergue comment le cadre d'analyse du Système National d'Innovation (S.N.I) reste incomplet pour apréhender les differentes structures de gouvernance de l'innovation. Enfin, pour que l'innovation puisse être un vecteur de développement inclusif et durable des économies les plus fragiles, il est nécessaire de renouveler les indicateurs prioritaires qui pilotent la recherche et la technologie. Trois axes structurent notre numéro spécial et portent sur la nécessité de renforcer les Capacités tout en améliorant les mécanismes de gouvernance de l'innovation.

ABSTRACT. This introductory article starts from the renewal of development challenges in Africa with regard to the increase in inequalities, climate change, food insecurity, population growth and political conflicts, and questions the effectiveness of public policies. In this context, it questions how to adapt a systemic and interactive framework for innovation to strengthen the societal capacities that drive new technological, economic and social trajectories. This proposal thus highlights how the framework for analyzing the National Innovation System (N.I.S) remains incomplete to understand the different governance structures for innovation. Finally, for innovation to be a vector of inclusive development and sustainable of the most fragile economies, it is necessary to renew the priority indicators that drive research and technology. Three axes structure our special issue and relate to the need to strengthen Capacities while improving the governance mechanisms of innovation.

MOTS-CLÉS. Politique d'innovation, Système Nationaux d'innovation, Système d'innovation agricole, Afrique, Transfert technologique, Développement inclusif.

KEYWORDS. Innovation policy, National system of innovation, Agricultural innovation system, Africa, Technology transfer, Inclusive development.

\section{Introduction Générale}

La concentration sur le continent Africain d'un nombre important de pays à revenus intermédiaires et moins avancés ${ }^{1}$ rend compte de l'échec ou de fortes incomplétudes des modèles de développement fondés sur des «transferts de trajectoires empruntés des pays dits développés » [EGG, 18]. En parallèle, ces économies sont exposées à de nouveaux enjeux sociétaux liés à des vulnérabilités créées par le changement climatique, l'insécurité alimentaire ou la croissance démographique exponentielle [DUR, 19]. Par ailleurs, les spécialisations productives sur les matières premières agricoles, minières, énergétiques privilégient des gouvernances rentières et les instabilités politiques inhérentes aux

\footnotetext{
${ }^{1}$ Sachant que la population d'Afrique subsaharienne devrait augmenter de 1,4 milliard d'habitants d'ici 2050, une poussée démographique sans précédent à l'échelle mondiale [MER,18].
} 
aspirations démocratiques ou aux conflits militaires extérieurs sont parfois fréquentes. La concentration géographique des aléas et les nouveaux enjeux précités interpellent les politiques publiques des pays de cette région, souvent orientées par les organismes internationaux afin de renouveler les objectifs du développement. Une proposition de ce renouvellement est reformulée par les Objectifs du Développement Durable (O.D.D) qui intègre notamment des objectifs de réduction des inégalités mondiales et des indicateurs pour le maintien de la biodiversité et la préservation de la capacité de choix des générations futures [GER,16].

Ce contexte international interroge l'évolution au sens donné au «développement» qui historiquement, est resté polarisé sur la promesse d'un rattrapage de pays industriels au regard d'indicateurs macro-économiques de richesse matérielle par la croissance du PIB et un pilotage par la compétitivité [UZU, 11]. L'illusion de cette promesse est aujourd'hui de plus en plus admise. La croissance contemporaine est connue comme renforçant les exclusions et les inégalités au sein des économies et entre les économies [STI,14]. Elle ne peut plus être associée à un développement « soutenable » pour tous. Un des mécanismes explicatifs est lié aux trajectoires technologiques dominantes qui sont devenues depuis la seconde guerre mondiale de moins en moins inclusives [SCH,17]. De plus, les externalités environnementales négatives sont croissantes et détruisent de manière accélérée les conditions d'existence de la vie à long terme [DAV,19]. Ce constat se solidifié par les travaux en Sciences Humaines et Sociales sur le développement depuis les années 1980. Il est aussi de plus en plus partagé par les organisations internationales [PLA,17] et contribue à réorienter les politiques publiques sur différentes échelles : nationales ${ }^{2}$ et internationales.

Cet état de fait exige un renouvellement des concepts et outils de pilotage du développement au sein duquel l'innovation est centrale et permet de transformer les trajectoires technologiques et sociétales permettant de créer une croissance ou un développement inclusif [DAN,17]. Dans cette perspective, sont interrogés les cadres d'analyse qui pilotent les investissements ou les soutiens à la science et la technologie. Au-delà d'une exigence d'inclusion sociale, les modes de production et de consommation de demain, et particulièrement ceux des pays les plus démunis, doivent aussi devenir résilients aux instabilités écologiques, socio-politiques croissantes. Ce renouvellement est également conceptualisé [LUN,16] et opérationnalisé auprès des organismes internationaux (OCDE, Banque Mondiale, FAO, IFPRI..., etc.) par le concept de Système d'Innovation qui se décline dans différentes acceptions d'usages possibles : national, sectoriel, local. Dans sa forme d'usage la plus courante (Système National d'Innovation), ce concept est un cadre d'analyse de la capacité des économies nationales à générer, mettre en œuvre, évaluer les transformations des modes de production et de consommation. Il structure depuis les années 2000, l'affirmation au sein des politiques publiques, d'un champ de gouvernance du développement par les politiques d'innovation [EDL,17, FAG, 18]. Néanmoins, les nouveaux enjeux liés aux S.N.I ne sont pas assez considérés sur le plan de la formalisation d'indicateurs à considérer pour mesurer l'innovation et l'apprentissage des économies en développement. De plus, dans une perspective internationalisée, les systèmes d'innovation sont constitués de réseaux d'acteurs et de contextes institutionnels qui soutiennent (ou entravent) la formation et la diffusion d'une innovation. Dans certains cas, ils peuvent être réductibles à des contextes territoriaux spécifiques, alors que dans d'autres, ils dépendent de stratégies d'acteurs, de réseaux et de dynamiques qui évoluent conjointement entre différentes parties du monde [BIN,17]. Ces éléments sont importants et non assez traduits dans la littérature en économie de l'innovation.

En référençant les cadres d'analyses systémiques de l'innovation dans différentes situations en Afrique francophone, ce numéro spécial de la revue Technologie et Innovation s'intéresse à la question suivante : En quoi la gouvernance des processus d'innovation est-elle tributaire des structures macro-

${ }^{2}$ «La croissance du PIB ne nous intéresse pas » Li Keqiang 2014 cité par Laurent 2017, p.29. 
économiques des économies considérées ? Ce numéro questionne ainsi la pertinence d'usage du cadre d'analyse mobilisé dans le contexte de pays peu industrialisés et laissés en marge des indicateurs classiques du développement. Il s'intéresse, dans ce contexte, aux problématiques d'adaptation de ce cadre d'analyse pour renforcer des capacités sociétales à gouverner de nouvelles trajectoires technologiques, économiques et sociales.

Les différentes études de cas localisées en Afrique francophone concernent des pays à revenus intermédiaires : Sénégal, Algérie, Cameroun et des Pays Moins Avancés (PMA) : Burkina Faso et Mali. Les points communs entre ces économies (identifiés par les différentes contributions du numéro), portent sur la structure des systèmes productifs, les fragilités de leur gouvernance institutionnelle et politique "d'Etats fragiles » [RAZ,17], et sans doute aussi, leurs ancrages historiques. Ces points communs convergent vers un objectif ou une ambition sur l'aspiration des populations à connaitre une amélioration rapide de leurs conditions de vie dans les décennies à venir, au regard de celles perceptibles dans les pays dits «développés ». Cette ambition interpelle la gouvernance plus globale des politiques d'innovation pour un développement socialement mieux partagé pour tous, entre les territoires et incluant les générations futures.

Au regard de cette ambition, nous proposons une synthèse des contributions apportées au prisme de trois questions transversales. Ces questions sont traitées dans les différentes contributions référencées dans ce numéro spécial par l'analyse des mécanismes de gouvernance de l'innovation et, de manière indirecte, des relations qui peuvent s'établir avec le développement économique.

Une première question peut être formulée de la façon suivante : En quoi le concept de «S.N.I » généré par l'économie évolutionniste et institutionnalisé par les organismes internationaux peut structurer les politiques d'innovation génératrices de nouvelles trajectoires de développement inclusives ? Ainsi un premier article de Casadella et Uzunidis rappelle les conditions d'émergence de ce concept dans les pays industriels puis son usage dans le contexte de pays émergents et moins avancés. Il explique comment ce cadre d'analyse se focalise sur les conditions qui permettent de générer puis de mobiliser dans leurs complémentarités les différentes ressources nécessaires à l'innovation (recherche, financement, capital humain.). Les institutions modelées par les politiques publiques initient des interactions entre acteurs du système et sécurisent les conditions d'usage de ces ressources, identifiées comme des «centralités » du processus systémique. Ainsi la défaillance des institutions, mais également l'informalité dominante d'une partie du système productif, ne créent pas les mêmes contextes d'opportunités pour les investissements entrepreneuriaux. Ce décalage conduit à proposer une déclinaison des S.N.I sur celui du renforcement de compétences et d'apprentissages comme un moyen d'agir sur la génération des institutions et des dynamiques entrepreneuriales. Un élément d'activation de ces systèmes repose sur la densification nécessaire des institutions éducatives dont les Universités sont une composante essentielle.

Un deuxième article de Le Bas et Nkakene Molou illustre et complète la disjonction précédemment constatée. Partant du besoin de mieux contextualiser la compréhension de l'innovation à la spécificité des pays faiblement industriels (Cameroun), il met en exergue la désynchronisation entre d'une part, l'offre de recherche scientifique et technique pour partie pilotée par des enjeux académiques, avec et d'autre part, la réalité des processus d'innovation activée par un secteur informel assez prépondérant. Il démontre ainsi que les modèles d'innovation portés par les pays industriels et qui focalisent l'objectif des S.N.I sur la mobilisation des investissements dans la recherche et la technologie sont peu adaptés. En symétrie, les modèles d'innovation mobilisés par les dynamiques socio-entrepreneuriales qui émergent du secteur productif informel sont focalisés sur des processus d'innovation pilotés par la recherche d'une efficacité supérieure dans la valorisation des ressources existantes (cognitives, techniques.). La "frugalité » du processus d'innovation induit donc sa dimension inclusive de situations de précarité. Elle est au centre de la capacité à innover dans les réalités étudiées [CAS, 15]. Un 
élément nécessaire de ces capacités renvoie cependant aussi, comme dans l'article précédent, à la formation de ressources humaines suffisantes.

Une seconde problématique proposée par ce numéro est la suivante : comment les structures des systèmes productifs concentrées sur une spécialisation dans le secteur primaire et plus particulièrement dans l'agriculture et l'agro-alimentaire, spécifient les conditions sectorielles de mobilisation des cadres d'analyses de l'innovation [SPI,09, FAU,18] ? En effet, « cause » ou « conséquence » des difficultés de développement des situations nationales étudiées (excepté le cas particulier de l'Algérie), la plupart des systèmes productifs est polarisée par la prééminence du secteur agricole et alimentaire. Cette spécialisation explique l'engouement pour le concept de Système d'Innovation Agricole (SIA) qui mobilise une littérature croissante différenciant plusieurs communautés d'usages [TOU,16]. Ce concept rend compte dans la littérature récente d'une hétérogénéité de situations tributaires de niveaux de développement, de contextes socio-politiques, et de la capacité des Etats à mettre en œuvre des politiques publiques socialement inclusives [GOU,19].

Ainsi, un article sur le Burkina Faso de Sawadogo Compaore, partant de travaux initiés par différents projets qui documentent les orientations des organismes internationaux comme l'OCDE ou la FAO, explique en quoi l'application du concept de Système d'Innovation à l'agriculture peut rendre compte des structures de gouvernance de l'innovation sectorielle. Il propose alors d'opérationnaliser par un prototype de guide méthodologique la caractérisation de ces dynamiques systémiques pour le pilotage des politiques d'innovation et de recherche.

Dans une démarche complémentaire, un article sur le Mali d'El Moctar analyse comment l'histoire et les projets de politique agricole et alimentaire permettent d'identifier les opportunités et les limites pour la conception et l'application d'une politique d'innovation sectorielle dans l'agriculture. Pour cela, il mobilise lui aussi le concept de Système d'Innovation et de Construction de Compétences.

Une troisième question se pose enfin comme suit : En quoi les promesses portées par l'économie de la connaissance, la formation du capital humain mais surtout la création de «capacités d'apprentissage » créent des opportunités pour l'activation des processus d'innovation ? L'hypothèse de départ est que les investissements dans ces domaines densifient et mettent en route des transferts technologiques de pays industriels ou émergents au bénéfice des transformations de systèmes productifs de pays plus fragiles.

Ainsi un article en sur l'Algérie d'Haudeville et Younes Bouacida souligne une situation paradoxale où des investissements publics élevés dans le capital humain et le système éducatif primaire et supérieur n'ont pas enclenché d'innovations technologiques et la croissance selon les prédictions des modèles de croissance endogène. L'explication principale de ce "paradoxe » que l'on retrouve également dans d'autres situations, porte sur le décalage entre la nature des investissements en capital humain basé sur des connaissances génériques, des savoirs académiques et la faiblesse de situations concrètes d'usages de ces connaissances afin de transformer ces savoirs en compétences et savoir-faire technologiques au sein de systèmes productifs locaux ${ }^{3}$.

Cette proposition explicative est illustrée par Casadella dans un article qui analyse dans le contexte du Sénégal de manière approfondie la dynamique d'innovation entrepreneuriale d'une industrie agroalimentaire majeure. En se référant au concept du Système d'Innovation et de Construction de Compétences, cette contribution, montre que si l'hybridation entre des savoirs académiques et tacites est une condition nécessaire à l'activation de la capacité d'innovation pour réaliser des transferts

\footnotetext{
${ }^{3}$ La faible densité intersectorielle d'un système productif très centré par sur la rente pétrolière est probablement aussi une contrainte majeure.
} 
technologiques nécessaires à la modernisation du système productif, elle reste insuffisante. Une condition de second ordre est focalisée sur l'adoption de technologies exogènes aux processus locaux d'innovation et l'existence d'opportunités d'investissements productifs que ce soit pour le secteur public ou privé ou de leur conjonction.

Ce débat sur les transferts de technologies rappelle d'ailleurs l'importance des environnements macro-économiques dans l'utilisation des nouvelles technologiques [TIG, 18 NUB, 19]. Ainsi dans cette thématique, la contribution de Nubukpo Kako s'intéresse de manière transversale aux nouvelles technologies liées à l'économie numérique en Afrique Francophone, qui peuvent être susceptibles de densifier les processus d'innovation dans l'agriculture et les mécanismes de participation citoyenne dans la formulation de politiques d'innovation et de recherche. Mais le numérique, tout en représentant des opportunités pour une meilleure condition d'accès à la connaissance ou une meilleure participation citoyenne à la gouvernance de politiques publiques, entraine également des menaces qui exigent des régulations institutionnelles. L'article nous rappelle que si le progrès technologique et les processus d'innovation portent sur des promesses de développement, leur réalisation au bénéfice de tous (y compris les générations futures) impose un réel engagement de l'action publique.

Sur le plan empirique, le renouvellement des cadres d'analyse systémique de l'innovation documente en l'espèce les structures institutionnelles de gouvernance des politiques d'innovation par le concept de S.N.I afin d'améliorer leur efficacité au regard des enjeux du développement. Ce concept porte une utilité heuristique pour décrire ce qui « devrait être »; il est aussi critiqué par les différentes contributions. Il est en effet élaboré dans les réalités institutionnelles de pays industriels où coexiste une diversité de modèles d'innovation liés à des structures productives spécifiques ancrées dans une société mondialisée et capitaliste, avec des infrastructures de recherche scientifique où l'innovation est pour partie gouvernée par des entreprises globalisées [BIN,17]. Dans le contexte de pays non industriels, deux difficultés majeures rendent difficile la mobilisation de ce concept pour une gouvernance effective des politiques d'innovation. La première est liée à la fragilité des institutions publiques en place, des ressources que peut mobiliser l'Etat, et des urgences de gouvernance prioritaires que fixent l'agenda public : crises alimentaires, instabilité des marchés internationaux, instabilités politiques voire militaires mais également des régulations macro-économiques souvent exogènes à ces économies. La deuxième est relative aux structures du système productif dominées par un secteur agricole et alimentaire encore prédominant et un secteur informel d'activités entrepreneuriales de petites entreprises imbriquées dans l'économie domestique. Cette structure productive génère une diversité de modèles d'innovations différente de celle des pays industriels. Elle est par ailleurs focalisée sur des objectifs de développement liés à des situations de précarité socio-économique d'une grande partie de la population. Les capacités de mobilisation des progrès scientifiques et technologiques qui exigent des conditions entrepreneuriales d'investissement (publiques ou privées) y sont beaucoup plus faibles que dans les pays industriels. La création d'une capacité politique nationale à mettre en système les ressources qui structurent l'innovation (connaissances, apprentissages, capital humain, financements, etc.) est alors dans ce contexte concentrée sur le secteur formel de l'économie. Elle se retrouve faiblement inclusive des réalités sociétales et entrepreneuriales dominantes caractéristiques de l'innovation dans les pays considérés.

Plusieurs solutions sont identifiables dans les travaux rassemblés par ce numéro spécial.

La première solution conduit à adapter le concept de S.N.I aux besoins de gouvernance des économies concernées en le focalisant sur des « ressources », ou des « innovations » motrices du point de vue de leurs impact intersectoriels. En termes de « ressources », le renforcement de compétences et de capacités d'apprentissages a été proposé comme central. En termes "d'innovation », le numérique est apparu sous certains conditions, vectrice de régulation et structurant des transversalités d'apprentissages. 
La deuxième concerne l'amélioration de la « sectorialisation » des conditions d'usage de la notion d'innovation, c'est-à-dire de mieux spécifier une opérationnalisation des conditions d'application du concept de Système d'Innovation sur des sous-composantes du système productif : agriculture, agroalimentaire... Elle impose de construire des guides méthodologiques adaptés à ces spécificités.

La troisième solution part de l'hypothèse que si le concept de S.N.I est faiblement adapté aux réalités structurelles des systèmes productifs et des demandes sociétales et politiques étudiées [TEM, 17], son application risque de ne bénéficier qu'aux composantes les plus visibles de ce système. L'intensification de transferts de technologies conçus pour de grandes entreprises et mis au point par les pays industriels présente des risques d'échecs structurels élevés. Ces transferts ne peuvent donc être considérés comme suffisants pour répondre aux besoins sociétaux et entrepreneuriaux du secteur informel. Au regard de cette hypothèse il est donc nécessaire d'interroger en quoi d'autres référentiels d'usage du concept de Système d'Innovation (SI) comme les Réseaux d'Innovation [DJE,18] ou autres dynamiques sectorielles [GAL,16] permettent de mieux structurer des cadres d'analyses des processus d'innovation et donc des politiques d'accompagnement adaptées aux réalités sociétales. Cette adaptation est tributaire de l'exigence de contribuer rapidement à la diminution de la pauvreté d'une population croissante. Cette condition est aussi une opportunité pour l'autoréalisation des processus d'innovation car elle densifie les conditions entrepreneuriales et les capacités d'investissement collectives dans l'articulation entre l'innovation technologique et l'innovation sociétale.

Une autre opportunité faiblement saisie par cette gouvernance des trajectoires technologiques est de mieux intégrer territorialement la transformation durable des ressources locales en biens et services qui améliorent les conditions de vie des populations de ces pays.

Plus globalement, ce numéro spécial met en exergue le fait que pour que l'innovation technologique soit un vecteur de développement inclusif durable des économies fragiles, il est nécessaire de renouveler les indicateurs prioritaires qui pilotent la recherche et la technologie [TEM, 18]. Cela semble primordial afin de substituer aux indicateurs qui se focalisent sur l'accroissent matériel de plus en plus obsolète, des indicateurs d'accroissement du bien être : éducation, santé, sécurité. Cela implique probablement des changements majeurs de gouvernance institutionnelle des politiques d'innovation et de recherche au sein même de pays industriels.

\section{Bibliographie}

[BIN,17] BINZ C., TRUFFER B., (2017), “Global innovation systems - a conceptual framework for innovation dynamics in transnational contexts", Research Policy (46), p. 1284-1298.

[CAS, 15] CASADELLA, V., LIU, Z., \& UZUNIDIS, D. (2015). Développement économique et capacités d'innovation dans la mondialisation (Vol. 1). ISTE Group.

[DAN, 17] DANIELS, C. U., USTYUZHANTSEVA, O., \& YAO, W. (2017). Innovation for inclusive development, public policy support and triple helix: perspectives from BRICS. African Journal of Science, Technology, Innovation and Development, 9(5), p. 513-527.

[DAV, 19] DAVIRON B. 2019. Biomasse une histoire de richesse et de puissance, Editions Quae.

[DJE, 18] DJELLAL F., GALLOUJ F.. (2018) «Les réseaux d'innovation public-privé dans les services (RIPPS) : une nouvelle expression des réseaux d'innovation dans une économie des services et du développement durable », Revue d'économie industrielle, vol. 161, no. 1, p 67-118.

[DUR, 19] DURY S, BENDJEBBAR P, HAINZELIN E, GIORDANO T, BRICAS N, eds. (2019) Food Systems at risk: new trends and challenges. Rome, Montpellier, Brussels, FAO, CIRAD and European Commission. DOI: 10.19182/agritrop/00080

[EGG, 18] EGG, J., GABAS, J. J., GÉRONIMI, V., \& VERNIÈRES, M. (2018). Essai sur l'économie politique du développement : en hommage à Philippe Hugon. Mondes en développement, (4), p. 137-164. 
[EDL, 17] EDLER, J., \& FAGERBERG, J. (2017). Innovation policy: what, why, and how. Oxford Review of Economic Policy, 33(1),p. 2-23.

[FAG, 18] FAGERBERG, J., LUNDVALL, B. Å., \& SRHOLEC, M. (2018). Global value chains, national innovation systems and economic development. The European Journal of Development Research, 30(3), p. 533-556.

[FAU, 18] FAURE G., CHIFFOLEAU Y., GOULET F., TEMPLE L., TOUZARD JM, (2018). Innovation et développement dans les systèmes agricoles et alimentaires. Edditions Quae, ISBN 9782759228126, 259 p.

[GAL, 16] GALLIANO D., NADEL S. (2016) « Les processus sectoriels de l'innovation environnementale : les spécificités des firmes agroalimentaires françaises », Économie rurale, vol. 356, no. 6, p. 47-67.

[GER, 16] GÉRARDIN, H., DOS SANTOS, S., \& GASTINEAU, B. (2016). Présentation. Des Objectifs du Millénaire pour le développement (OMD) aux Objectifs de développement durable (ODD): la problématique des indicateurs. Mondes en développement, (2), p. 7-14.

[GOU, 19] GOULET F., LE COQ JF., SOTAMAYOR O., (2019). Sistemas y políticas de innovación para el sector agropecuario en América Latina. GOULET F. (ed.), Le Coq, Jean-François (ed.), Sotomayor, Octavio. Rio de Janeiro : E-papers, 23-56. ISBN 978-85-7650-598-3

[LAU, 17] LAURENT E. Notre bonne fortune - Repenser la prospérité (PUF, 2017)

[LUN, 16] LUNDVALL, B. A. (2016). Innovation systems and development: history, theory and challenges. In Handbook of Alternative Theories of Economic Development. Edward Elgar Publishing.

[MER, 18] MERCANDALLI, S. LOSCH B. (2018) Une afrique rurale en mouvement. Dynamiques et facteurs des migrations au sud du Sahara. Rome, FAO et CIRAD , 60 p.

[NUB, 17] NUBUKPO K., (2019). L'urgence africaine : changeons le modèle de croissance. Editions Odile Jacob, Paris $234 \mathrm{p}$.

[PLA, 17] PLANES-SATORRA S., PAUNOV C. Inclusive innovation policies: Lessons from international case studies. OECD Science, Technology and Industry Working Papers, 2017 (2), 1.

[RAZ, 17] RAZAFINDRAKOTO, M., ROUBAUD, F., WACHSBERGER, J.-M., ROUBAUD, F., \& WACHSBERGER, J.-M. (2017). L'énigme et le paradoxe: économie politique de Madagascar. Marseille: [Paris]: IRD éditions ; AFD, Agence française de développement.

[SCH, 17] SCHILLO, R. S., \& ROBINSON, R. M. (2017). Inclusive innovation in developed countries: The who, what, why, and how. Technology Innovation Management Review, 7(7).

[SPI, 09] SPIELMAN, D. J., EKBOIR, J., \& DAVIS, K. (2009). The art and science of innovation systems inquiry: Applications to Sub-Saharan African agriculture. Technology in society, 31(4), p. 399-405.

[STI, 14] STIGLITZ JE, GREENWALD B., La nouvelle société de la connaissance, Éditions Les Liens qui libèrent, Paris, 2014.

[TEM, 18] TEMPLE L., BARRET D., BLUNDO CANTO G., DABAT MH., DEVAUX-SPATARAKIS A., FAURE G., HAINZELIN E., MATHÉ S., TOILLIER A., TRIOMPHE B (2018)., « Assessing Impacts of Agricultural Research for Development: a systemic model focusing on outcomes », Research Evaluation, vol. 27, n² 2, p. 157-170

[TEM, 17] TEMPLE L., MACHIKOU NDZESOP N., FONGANG FOUEPE G.F., NDOUMBE NKENG M., MATHE S., (2017) « Système national de recherche et d'innovation en Afrique : éclairage du Cameroun », Innovations, $\mathrm{n}^{\circ}$ 53, p.41-67

[TIG, 18] TIGABU A.D. (2018). Analysing the diffusion and adoption of renewable energy technologies in Africa: The functions of innovation systems perspective. African Journal of Science, Technology, Innovation and Development, 10(5), p. 615-624.

[TOU, 15] TOUZARD J.M, TEMPLE L., FAURE G., TRIOMPHE B., (2015) Innovation systems and knowledge communities in the agriculture and agrifood sector: a literature review. Journal of Innovation Economics and Management, 2 (17): p. 117-142.

[UZU, 11] UZUNIDIS, D., LAPERCHE, B., (2011) « The new mercantilism and the crisis of the global knowledge economy », Journal of the Knowledge Economy, vol. 2, n³, 2011. 